\title{
L'immigration d'ex-URSS et les colonies de Cisjordanie et de
} Gaza

In: Revue européenne de migrations internationales. Vol. 16 №3. pp. 201-218.

Citer ce document / Cite this document :

Berthomière William. L'immigration d'ex-URSS et les colonies de Cisjordanie et de Gaza. In: Revue européenne de migrations internationales. Vol. 16 N³. pp. 201-218.

doi : $10.3406 /$ remi.2000.1750

http://www.persee.fr/web/revues/home/prescript/article/remi_0765-0752_2000_num_16_3_1750 


\title{
L'immigration d'ex-URSS et les colonies de Cisjordanie et de Gaza
}

\author{
William BERTHOMIÈRE*
}

«La frontière se trouve là où habitent des juifs, et non pas sur un tracé de carte » déclarait Golda Meir, alors Premier ministre d'Israël, lors d'une cérémonie organisée pour de nouveaux immigrants soviétiques sur le plateau du Golan'. Vingt années plus tard, le 14 janvier 1990, un autre Premier ministre, Yitshak Shamir, affirmait face à l'éveil migratoire post-1989 qu' "une grande immigration nécessite un grand Israël ». Ces deux déclarations soulignent avec force la pérennité du lien étroit qu'a su tisser Israël entre migration et stratégie territoriale. Né de l'aliya l'immigration juive -, Israël a toujours soutenu l'idée que l'entrée de nouveaux immigrants représentait une force vive permettant d'assurer sa sécurité et ses options géostratégiques ${ }^{2}$. Cette dimension de l'immigration s'est trouvée renforcée au lendemain de la Guerre des Six-jours alors que le choix de conserver les territoires conquis s'affirmait.

Au cours des vingt dernières années, la question démographique a pris une place de plus en plus centrale dans le conflit israélo-palestinien, soulignée notamment par l'occurrence dans le milieu scientifique d'expressions comme : « guerre des berceaux », « bombe démographique » ou encore «bataille de chiffres ». Ainsi, au moment fort qu'est la première Intifada ${ }^{3}$, l'idée-force qui fait de la démographie « la plus imparable des armes palestiniennes » interpelle vivement les gouvernants israéliens. Le rapport de force, opposant traditionnellement le dynamisme naturel de la population palestinienne au renfort démographique constitué par la diaspora juive, tend en effet à s'incliner en faveur des Palestiniens : la courbe de l'immigration juive se

* Chargé de recherche CNRS, Équipe MIGRINTER (Migrations Internationales : Espaces et Sociétés), Poitiers.

1 Citation de l'article publié dans le journal Maariv (Tel Aviv, 26 septembre 1971) faite par Kapeliouk (1975:21).

2 Voir notamment l'ouvrage de Tom Segev, Les premiers Israéliens, Paris, Calmann-Levy, 1998.

3 De décembre 1987 à 1991. 
maintient globalement à un faible niveau ponctué de quelques sursauts migratoires ${ }^{4}$ et le solde migratoire annuel est même par trois fois négatif sur cette période ${ }^{5}$.

En ouvrant la voie à l'émigration juive d'ex-URSS, l'effondrement du bloc soviétique a apporté un net renouveau migratoire à Israël (figure 1) et fait glisser la crainte d'une modification des rapports de force dans le « camp » palestinien car jusqu'à cet événement, les capacités israéliennes de colonisation des territoires occupés s'étaient progressivement réduites aux seules migrations « internes » ${ }^{6}$. Les événements de 1989 et leurs répercussions migratoires ont donc été suivis avec le plus grand intérêt par Yasser Arafat et les leaders palestiniens ${ }^{7}$; Abu Mazen ${ }^{8}$ allant même jusqu'à déclarer :

Figure 1 : L'immigration en Israël suivant les continents d'origine (1948-1999)

Source : Central Bureau of Statistics, Jerusalem, 2000.

4 Les sursauts enregistrés dans la courbe de l'immigration venue d'Europe sont principalement liés à des périodes de détente de la politique soviétique concernant l'émigration juive.

5 Respectivement - 4 700, - 4800 et - 1100 en 1985, 1986 et 1988.

6 Le terme de migration interne est employé uniquement par opposition à celui de migration internationale afin d'illustrer la configuration migratoire. Sur le plan géopolitique, ces mobilités correspondent à un mouvement de colonisation.

7 Depuis toujours, les leaders politiques de l'OLP ont pris la mesure de l'impact géostratégique que représentait l'éventualité d'une libéralisation de l'émigration en URSS. Au cours des années soixante-dix, les rapprochements entre Soviétiques et Américains, qui ont toujours eu pour conséquence - voire préalable - l'émigration de milliers de juifs soviétiques, constituaient une source d'inquiétudes au sein des milieux palestiniens. En réponse à la détente Est-Ouest, les opérations anti-israéliennes de la branche arméc de l'OLP avaient d'ailleurs pris comme objectif les émigrants juifs d'ex-URSS. Fin septembre 1973, alors que la guerre du Kippour est sur le point d'éclater, Golda Meïr, Premier ministre de l'époque, avait dû se rendre en Autriche suite à l'attaque d'un train transportant des juifs quittant l'URSS (Bensimon, $1989: 233$ ).

8 Secrétaire général du Comité exécutif de l'OLP. 
«Pour comprendre le danger que représente l'immigration, nous devons nous rappeler que lorsqu'Israël conquit $78 \%$ de la Palestine, il ne comptait que 600000 résidents. C'est alors que l'immigration se mit en marche d'Irak, du Yémen, d'Égypte et du Maroc pour combler le déficit. Je suis sûr que, si les effectifs étaient restés ceux du passé, Israël n'aurait pas survécu. Pour Israël, l'immigration est comme une artère reliée au cour d'un homme : elle nourrit l'économie, la troupe, la main-d'auvre et la paysannerie. C'est pourquoi nous considérons qu' elle représente le principal défi que la nation arabe ait à affronter » (Fargues, $1975: 73$ ).

La question de l'immigration récente venue d'ex-URSS a donc rapidement pris une dimension géopolitique, renforcée par la prompte publication d'articles scientifiques sur ce thème. Dès 1990 , The Journal of Palestine Studies (vol. XIX, $n^{\circ} 4$ ) publie un article de Geoffrey Aronson intitulé : « Soviet Jewish Emigration, the United States, and the Occupied territories » qui souligne la tonalité stratégique de cette immigration ${ }^{9}$. Un an plus tard, la LIDLP ${ }^{10}$ fait paraître dans un ouvrage sur la «question palestinienne et le droit international » une des toutes premières recherches en français sur l'immigration des Juifs soviétiques en termes de géopolitique et l'année suivante le Journal of Palestine Studies propose une bibliographie spéciale de plus de 300 titres sur les « juifs d'ex-URSS et Israël ${ }^{11}$.

Après plus d'une décennie d'immigration juive d'ex-URSS et alors que le processus de paix marque le pas et cède la place à une seconde Intifada, il convient d'observer dans les faits le rôle joué par cette immigration puisque le débat se recentre aujourd'hui autour de la dimension démographique du conflit ${ }^{12}$.

\section{La place des Territoires occupés dans la géographie de l'immigration d'ex-URSS}

Depuis l'effondrement du bloc soviétique, Israël a ouvert ses frontières à plus de 800000 ex-Soviétiques ${ }^{13}$. Ces immigrants, dont plus d'un tiers est entré au cours des deux premières années de la vague migratoire (figure 1), ont élu résidence dans les

9 Geoffrey Aronson est le rédacteur du Report on Israeli Settlement in the Occupied Territories, publication bimensuelle de la Foundation for Middle East Peace (http://www.fmep.org).

10 Ligue internationale pour le droit et la libération des peuples. Dans cet ouvrage intitulé Le dossier Palestine : la question palestinienne et le droit international (Paris : La découverte, 1991), l'immigration des juifs soviétiques est traitée comme chapitre à part entière dans la seconde partie consacrée aux « Droits nationaux et droits politiques. Obstacles et conditions d'application ».

11 Leenah Al-Qattan, «Soviet Jews and Israel : Immigration and settlement. A selected bibliography, 1985-October $1992 »$, Journal of palestine Studies, XXII, n 2 (Winter 1993), pp. 125-144.

12 En France, cette question a donné lieu à un récent débat organisé par l'INED et intitulé « L'arrière plan démographique de l'explosion de violence en Israël-Palestine » (Jeudi 30 novembre 2000).

13 Pour plus d'informations, voir le numéro spécial « Nouveaux visages de l'immigration en Israël » de la Revue Européenne des Migrations Internationales (REMI), vol. 12, n 3, 1996. 
principaux districts israéliens : ceux de Tel Aviv, du Centre et de Haïfa. Aux premières heures de l'immigration, la conjugaison d'une politique d'accueil dite "d'intégration directe »- laissant le libre choix du lieu de résidence - et d'une forte culture urbaine du côté des immigrants - notamment chez ceux venus de Russie et d'Ukraine - a en effet contribué à dessiner une géographie de l'immigration particulièrement polarisée autour de Tel Aviv (et ses villes satellites) et de Haïfa, puis dans une moindre mesure de Jérusalem et de Be'er Sheva.

À cette période, les Territoires occupés sont en marge des choix résidentiels des ex-Soviétiques, la Cisjordanie et Gaza ne regroupant qu'une faible part des immigrants. Au lendemain de la grande vague migratoire de 1990-1991, seul 1,2\% des immigrants d'ex-URSS réside au-delà de la ligne verte ${ }^{14}$. Au fil des années, les choix résidentiels se sont modifiés sous l'influence des difficultés d'accès au logement (saturation des capacités d'accueil dans les principales localités d'Israël et flambée des prix des logements, à l'achat comme à la location). La politique publique de soutien à la construction et à l'installation de population dans les espaces périphériques du pays (Galilée et Néguev) ainsi que dans les territoires conquis depuis 1967, a contribué fortement à ce phénomène.

Figure 2 : Immigrés de l'ex-URSS selon leur lieu de résidence (en \%)

Source : Central Bureau of Statistics, Immigrant Population from USSR (Former), selected data 1998, Jerusalem, 200, p.30.

14 Hors Jérusalem-Est. La ligne verte délimite l'ancienne frontière israélo-jordanienne (19491967). 
À la fin des années quatre-vingt-dix, cette politique semble avoir, en partie, porté ses fruits : le district Sud étant devenu le premier lieu de résidence des exSoviétiques (figure 2 et carte 1) (Berthomière, 1998 ; 2000). Les transformations de leur répartition s'expliquent par le développement des migrations internes et la corrélative réorientation des filières migratoires, qui semble avoir " profité » aux Territoires occupés. Sur la période 1990-1998, la proportion de nouveaux immigrants d'ex-URSS s'installant à Gaza et en Cisjordanie a plus que doublé, passant de 0,8\% à $1,9 \%$ (figure 2). Les mobilités internes ont en effet renforcé la place des Territoires occupés dans la géographie de l'immigration d'ex-URSS : pour exemple, si pour les ex-Soviétiques immigrés entre 1990 et 1991, la part de ceux installés au-delà de la ligne verte était d'environ $1 \%$, celle-ci avait plus que doublé en 1998 (l'effectif étant passé de 4000 à 6600 , figures 2 et 3).

Globalement, ces recompositions migratoires ont abouti à l'installation de plus de 15350 ex-Soviétiques en Cisjordanie et à Gaza (hors quartiers de Jérusalem-Est). Comparé à la population israélienne « de longue date », il ressort que le contingent exsoviétique est proportionnellement deux fois moins représenté dans les Territoires occupés (le ratio brut de population s'établissant autour de 3,75 pour 1,80). Cependant, il convient de dépasser ces données de cadrage et de replacer cette immigration dans les contextes politique et géostratégique des colonies israéliennes en observant plus précisément le cas de la Cisjordanie, puisqu'elle regroupe la quasi-totalité des exSoviétiques au-delà de la ligne verte, et celui de Jérusalem.

Figure 3 : Ex-Soviétiques résidant en Cisjordanie et à Gaza en 1998 selon leur année d'immigration

\section{Illustration non autorisée à la diffusion}

Sources : Central Bureau of Statistics, Immigrant Population from USSR (Former), selected data 1998, Jerusalem, 2000 , p.37. 
Carte 1 : Principales villes de résidence des ex-Soviétiques (1998)

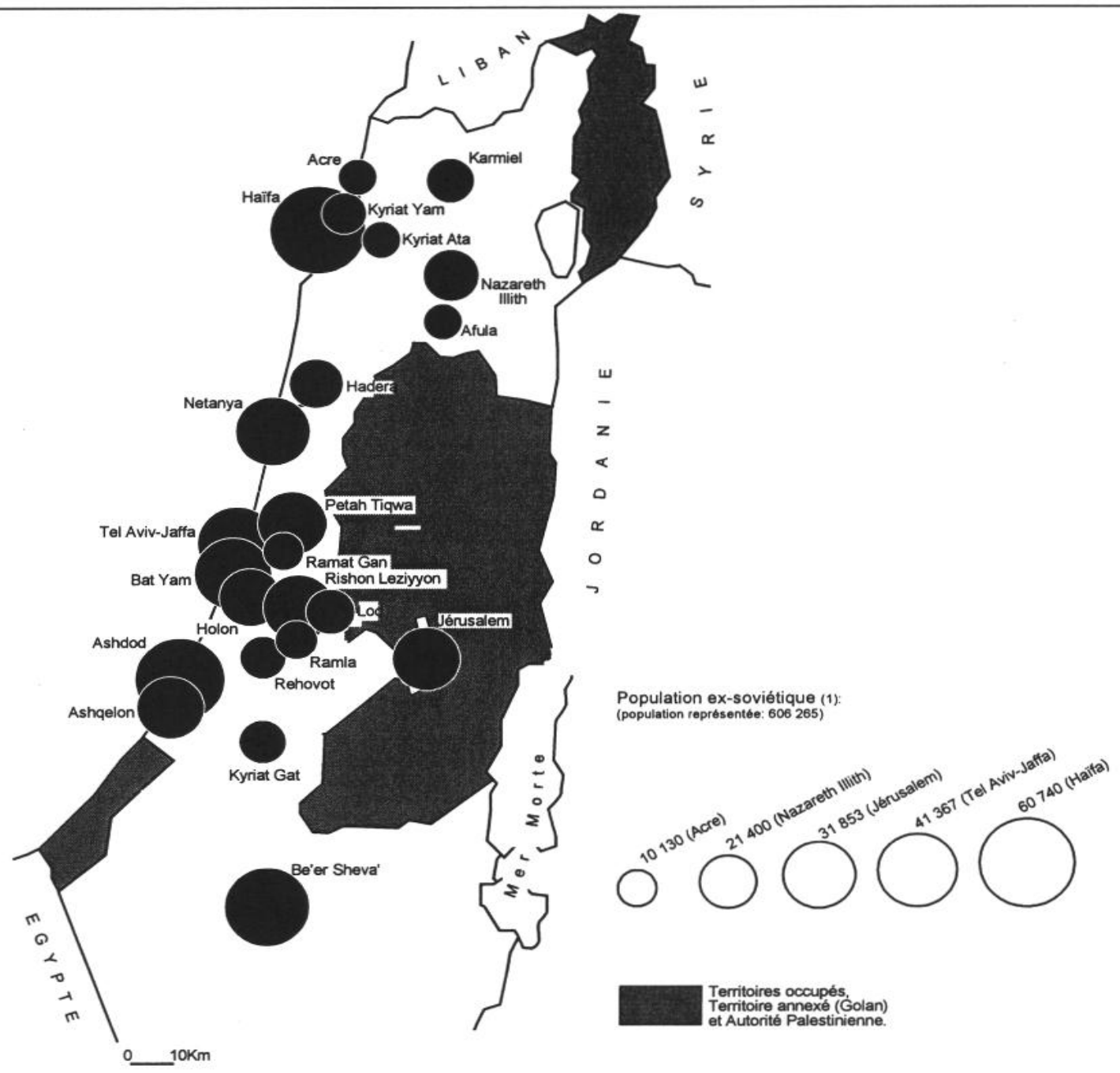

(1): Seules sont représentées les villes regroupant plus de 10000 immigrants au 30 septembre 2000

\section{Une représentation des colonies de Cisjordanie et de Gaza teintée d'《 utilitarisme »}

Les colonies juives de Cisjordanie et de Gaza ont connu depuis ces dernières années une nette croissance de population grâce aux mouvements sionistes religieux comme le Gouch Emounim ${ }^{15}$ mais aussi de par les mouvements internes de

15 Le Gouch Emounim ou «Bloc de la foi », groupe de sionistes-religieux créé au lendemain de la Guerre du Kippour, prône au nom d'un «messianisme territorial » l'implantation dans les territoires afin "d'accélérer le processus de rédemption ». Voir notamment DIECKHOFF (A.), 1993, L'invention d'une nation. Israël et la modernité politique, Paris, Gallimard (nrf essais), 352 p. et ATTIAS (J.-C.), BENBASSA (E.), 1998, Israël imaginaire, Paris, Flammarion, (essais), 391 p. 
déconcentration, liés au départ des populations des métropoles israéliennes vers les périphéries de ces villes.

Ces mobilités, impulsées principalement par les faveurs fiscales et sociales offertes par l'État pour toute installation au-delà de la ligne verte, ont fait des Territoires occupés l'un des deux seuls espaces attractifs (avec le district central) présentant une balance migratoire positive $:+36000$ (carte 2). En effet, une large part de ces colonies constitue aujourd'hui la grande couronne des banlieues des deux principales agglomérations israéliennes que sont Tel Aviv et Jérusalem. Avec l'avènement du Likoud (droite nationaliste) au pouvoir en 1977, la politique de colonisation, définie par Menachem Begin et Ariel Sharon a été mise en place en deux temps. La colonisation a reposé d'abord sur un mouvement idéologique articulé sur le mythe du «Grand Israël ». Puis, conscients de l'épuisement à court terme de ce courant, les dirigeants israéliens ont mis sur pied une politique d'aides publiques destinées à drainer vers elles la population juive : larges offres de logements subventionnés dans des colonies aisément accessibles depuis Tel Aviv et Jérusalem grâce à la création d'une importante infrastructure routière ${ }^{16}$. Cette politique, lancée dès 1981 , s'est poursuivie au cours des années quatre-vingt-dix sous l'ensemble des différents gouvernements.

Au moment où se libéralise l'émigration juive en ex-URSS, les colons juifs de Cisjordanie et de Gaza étaient 72800 (dont $95 \%$ en Cisjordanie) répartis dans 81 implantations, et, aujourd'hui, leur nombre s'établit autour de 200000 (96,5\%) dans près de 150 colonies, soit un taux de croissance brute de population de $174 \%{ }^{17}$. Quelle a été la participation des ex-Soviétiques dans cette croissance sur la période 1989$2000^{18}$ ?

En ce qui concerne la bande de Gaza, l'image globalement négative que revêt cet espace chez les immigrants d'ex-URSS a naturellement limité leur participation à la croissance démographique des colonies. Seules 8 des 16 implantations de Gaza ont accueilli des ex-Soviétiques et seulement 3 d'entre elles ont vu l'entrée de plus de 10 immigrants : Alei Sinai, Rafiah Yam et Neve Dekalim ${ }^{19}$ (carte 3). Cette dernière, qui est la plus importante des trois ( 285 hab.), a totalisé 34 entrées, ce qui représente

16 Pour exemple, selon une étude menée par le Palestinian Center for Human Rights (PCHR) intitulée : "A comprehensive survey of Israeli settlement in the Gaza strip (series study $n^{\circ} 10$ ) 》 (disponible sur internet : www.pchrgaza.org), le coût d'acquisition d'un duplex de $70 \mathrm{~m}^{2}$ avec 2 chambres à Neve Dekalim est de $50121 \mathrm{US} \$$ alors qu'il serait au moins du double en Israël. De plus, le taux de crédit associé à l'achat est moindre (env. 4\%) et une prime - d'un montant situé entre un quart et un tiers de la valeur d'achat - est offerte si le propriétaire reste quinze années à Gaza.

17 Ces données n'intègrent pas la population juive de Jérusalem-Est.

18 Les données utilisées couvrent la période du 1.1.1989 au 30.9.2000.

19 La colonie de Neve Dekalim (créée en 1983) est la plus importante des implantations de Gaza et elle constitue le centre administratif du Gush Katif (Bloc du Sud) : ensemble de colonies installées sur des terres appartenant aux villes palestiniennes de Khan Younis et Rafah. 
$16 \%$ de l'immigration globale enregistrée sur la période. Pour Alei Sinai et Rafiah Yam, la situation est toute autre car les ex-Soviétiques constituent la presque totalité de l'immigration bien que celle-ci reste très modeste : 11 migrants pour chacune sur la décennie analysée. Replacés dans le contexte de la colonisation de Gaza, ces faibles effectifs soulignent le fait que les implantations y sont essentiellement structurées autour du « religieux », ce qui répond aux attentes d'une très faible portion des immigrants d'ex-URSS, majoritairement laïques.

Le bilan pour la Cisjordanie est sans commune mesure avec celui de Gaza car le contingent ex-soviétique y est presque totalement regroupé, excédant les 15000 immigrants. Afin d'appréhender dans son ensemble cette dynamique migratoire, il convient de mettre en phase l'immigration et les types de colonies qui ont été créées. Pour cela, rappelons que la Cisjordanie a vu la création de dizaines de colonies aux objectifs stratégiques multiples, principalement le containment des zones de peuplement palestinien et la rupture de leur continuité ${ }^{20}$.

À travers ce prisme d'analyse, il ressort que les cinq premiers lieux d'implantation des ex-Soviétiques (rassemblant à eux seuls plus des deux tiers de la vague migratoire) intéressent seulement trois grands types de colonies : en premier lieu, les colonies adjacentes à la ligne verte et constituant une zone tampon avec Israël ${ }^{21}$ comme Ma'aleh Adoumim (3 200 ex-Soviétiques), celles ancrées au cœur de la Cisjordanie avec pour but de rompre la continuité territoriale comme Ariel (6 000), Karne Shomeron (620) ou Givat Zeev (400) et enfin, les colonies de contrôle territorial situées au cœur même des espaces de forte concentration palestinienne avec Kyriat Arba (1060; carte 3).

D'un point de vue global, ces premiers résultats soulignent que les immigrants ex-Soviétiques ont choisi de s'implanter majoritairement dans les plus importantes colonies de Cisjordanie puisque ces cinq implantations font partie du groupe des neuf colonies urbaines ${ }^{22}$, qui rassemblent plus de $50 \%$ des colons ${ }^{23}$. À une échelle plus fine de réflexion, ces choix témoignent d'une représentation de la géographie des colonies teintée d'《 utilitarisme ». La proximité des bassins d'emplois de Tel Aviv (pour Karne Shomeron et Ariel) et de Jérusalem (pour Givat Zeev et Ma'ale Adoumim) semble en effet expliquer ces localisations. Nos propres enquêtes réalisées à Ariel avaient souligné cette dimension. Pour exemple, un jeune immigrant venu de Gomel, installé comme chauffeur de taxi, nous avait souligné qu'Ariel apparaissait aux yeux des exSoviétiques comme une « cité dortoir de qualité » à moins de quarante minutes de la banlieue de Tel Aviv et que la dimension idéologique n'était que mineure dans leur choix d'installation. Notre insistance sur l'enjeu politique d'un tel choix de résidence avait très vite provoqué une réaction de protestation des collègues du nouvel

20 Pour une étude approfondie de cette question, voir notamment Dieckhoff (1987).

21 Ce type de colonies relève d'un double objectif : renforcer la présence juive sur la ligne verte et séparer les Arabes israéliens des Palestiniens de Cisjordanie.

22 Le terme hébreu toshava définit ces centres résidentiels.

23 Les quatre autres sont : Immanuel, Modi'in Illith (Kyriat Sefer), Betar Illith et Efrat. 
Carte 2 : Migrations internes en Israël par district d'origine (1984-1990)

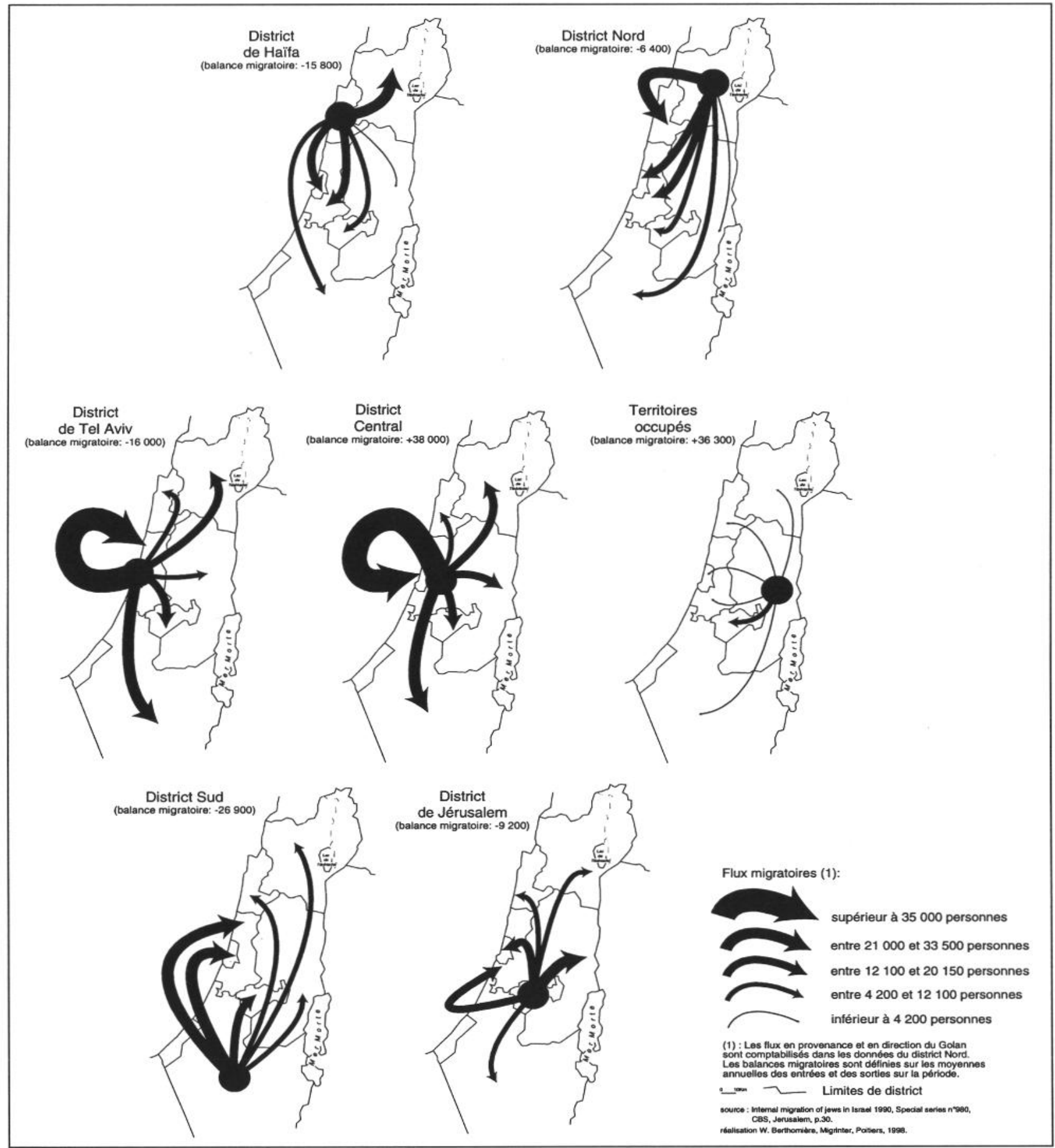

immigrant. Ces derniers avaient insisté sur le fait qu'ils n'étaient pas des colons mais des artisans peu fortunés qui préféraient - à coût égal - résider dans une maison de $100 \mathrm{~m}^{2}$ à Ariel plutôt que dans un deux pièces de Tel Aviv. Même si, indéniablement, les immigrants ont conscience des répercussions politiques de leur installation au-delà de la ligne verte, leur représentation de l'espace est avant tout tournée vers la quête d'un lieu où est optimisé au maximum le rapport qualité de vie/intégration 
socioprofessionnelle. Ces arguments nous ont été réaffirmés avec encore plus d'intensité lors d'entretiens réalisés à Ma'ale Adoumim.

Que nous ne retenions pour l'analyse que les plus peuplées des colonies ou que nous les considérions dans leur ensemble, l'existence d'une réelle motivation idéologique semble donc peu avérée dans les choix d'installation car une large part de l'effectif ex-soviétique se répartit dans des colonies non religieuses, principalement à Ariel et à Ma'ale Adoumim, cités-dortoirs de Tel Aviv et Jérusalem (figure 4).

Figure 4 : Immigration totale et immigration d'ex-URSS dans les principales colonies urbaines de Cisjordanie, selon le type de colonies (période du 1.01.1989 au 30.09.2000)

Source : ministère israélien de l'Immigration, 2000.

Parmi les implantations « religieuses », seule Kyriat Arba, à proximité d'Hébron, a accueilli une communauté ex-soviétique conséquente (environ 1000 immigrants soit près de $19 \%$ de la population totale de la colonie (carte 3 et figure 4). L'importance de ce contingent ex-soviétique s'explique également par le fait que Kyriat Arba est un haut lieu du nationalisme juif où déjà dans les années soixante-dix et quatre-vingts des Refuzniks avaient symboliquement choisi de s'établir (Dieckhoff, 1996 : 179-180). Les autres colonies ayant reçu un nombre d'immigrants constituant plus d'un cinquième de la population totale sont Teqoa $(21,3 \%)$, Noqedim $(22,4 \%)$, Barqan $(21,7 \%)$ et Yitav $(35,5 \%)$. Pour les trois premières, leur localisation laisse supposer des motivations similaires à celles décrites pour Ariel et Ma'ale Adoumim, seule la dernière, située dans la vallée du Jourdain soulève l'interrogation. L'histoire de cette colonie apporte vraisemblablement la réponse puisqu'elle a été créée par des immigrants soviétiques venus avec la vague des années soixante-dix ; le choix d'y 
Carte 3 : Principales colonies d'installation des ex-Soviétiques en Cisjordanie et à Gaza (1989-2000)

(c) William Berthomière, Isabelle Bouhet - Migrinter - 2000. 
résider s'appuie donc, selon toute vraisemblance, sur un réseau migratoire à base familiale ${ }^{24}$. De plus, l'observation de la répartition de la quarantaine de colonies regroupant plus de 30 ex-Soviétiques laisse apparaître un fort regroupement le long de la ligne verte et dans le «Grand Jérusalem »; les seules exceptions étant la colonie d'Eli et celle de Ma'ale Ephraim avec respectivement 220 immigrants (soit 19,7\% de la population de la colonie) et 275 immigrants (soit 16,5\%, cf. carte 3 ).

Il ne s'agit en aucun cas de minorer l'impact stratégique de ces choix d'implantation en arguant des nécessités liés à l'insertion socioprofessionnelle des nouveaux immigrants. L'affirmation de ces motivations d'ordre économique n'a pu se faire que parce qu'il existait, parallèlement, une politique gouvernementale d'incitation à l'établissement au-delà de la ligne verte affirmée avec force : celle-ci s'étant d'ailleurs maintenue en dépit des engagements pris lors des différentes négociations sur le processus de paix qui stipulaient le gel des constructions. Le maintien de cette politique, alors que les moyens qu'elle accapare auraient pu être exploités pour accroître les capacités d'accueil en Galilée et dans le Néguev, n'a eu pour effet que de repousser encore plus vers les colonies les ex-Soviétiques qui avaient choisi de résider dans les districts de Tel Aviv et Jérusalem. L'observation des migrations internes souligne avec clarté la forte propension des ex-Soviétiques à délaisser les districts de Tel Aviv et de Jérusalem au profit de la Cisjordanie ${ }^{25}$, principalement Ma'ale Adoumim, Ariel et la kyrielle de colonies sises dans le «Grand Jérusalem » (cf carte 3). Rappelons simplement qu'en 1997, les 3/4 des transactions immobilières réalisées à Ariel étaient le fait de nouveaux immigrants ${ }^{26}$. Replacée dans le contexte stratégique d'une colonie créée dans l'objectif de fractionnement de la «Samarie », Ariel trouve donc un soutien de premier ordre dans les mobilités internes des exSoviétiques.

\section{Jérusalem, « un symbole oublié... »}

Située au cœur de ce réseau de colonies, Jérusalem cristallise toutes les tensions que soulèvent ces géostratégies où s'opposent les démographies juive et palestinienne. Poursuivons donc notre analyse par la mesure de l'impact démographique de l'immigration ex-soviétique.

Au lendemain de la victoire de 1967, la Knesset (le parlement israélien) promulgue une loi qui annexe la partie orientale de la ville et fait de celle-ci la capitale « unifiée »d'Israël (cf. encadré). À partir de cette date, un vaste programme de développement de quartiers juifs au Nord, au Sud et à l'Est de Jérusalem est mis sur

24 La faiblesse de la population totale de la colonie (110 personnes) semble confirmer cette hypothèse.

25 En 1995, la comparaison des balances migratoires des immigrants d'ex-URSS et de la population juive pour la Cisjordanie et de Gaza était très en faveur des premiers : les taux s'établissant à $88,1 \%$ contre $43,9 \%$.

$26 \mathrm{Ha}^{\prime}$ aretz Internet English edition, June 16, 1999. 
pied dans le but d'affirmer la souveraineté juive sur l'ensemble de la municipalité afin de prévenir une éventuelle partition de la ville (Newman, $1992: 40)^{27}$.

\section{Jérusalem, 1949-2000 : Une succession de résolutions et de lois}

Le 9 décembre 1949 (résolution 303) : «L'ONU réaffirme « son intention de voir instaurer à Jérusalem un régime international permanent et confirme «expressément les dispositions suivantes de la résolution 181 (II) : 1) la ville de Jérusalem sera constituée en corpus separatum sous un régime international spécial et sera administrée par les Nations Unies, 2) le conseil de tutelle sera désigné pour assurer les fonctions d'Autorité chargée de l'administration, 3) la ville de Jérusalem comprendra la municipalité actuelle de Jérusalem, plus les villages et centres environnants, dont le plus oriental sera Abu Dis, le plus méridional Bethléem, le plus occidental Ein Kerem (y compris l'agglomération de Motsa) et le plus septentrional Shu'afat ».

Dans la ligne de la pensée de Ben Gourion qui déclarait le 12 décembre : " Jérusalem est une partie inséparable d'Israël et sa capitale éternelle. Aucun vote des Nations Unies ne pourra modifier ce fait historique », le 13 décembre, la Knesset vote une loi qui proclame Jérusalem-Ouest capitale d'Israël.

Le 27 juin 1967: «par l'amendement 11 à la loi 5727, la Knesset décrète que « la loi, la juridiction et l'administration de l'Etat s'étendront à toute portion d'Eretz Israël désignée par décret gouvernemental. »

Le 28 juin 1967 : « le gouvernement fait de Jérusalem-Est : « une région où la loi, la juridiction et l'administration d'Israël sont en vigueur »

Le 30 juillet 1980 : " la Knesset adopte une loi fondamentale qui proclame que : « Jérusalem entière et unifiée est capitale d'Israël... l'État accordera la priorité à Jérusalem pour ce qui concerne son développement ».

Le 27 novembre 2000 : « la Knesset adopte une loi sur les frontières de Jérusalem. Celle-ci stipule que tout transfert d'autorité sur la ville à une entité non-israélienne ou toute modification des limites municipales doit être soumis à la Knesset et obtenir une majorité de 61 députés pour être validé ».

Sources : RIVIERE-TENCER (V.), ATTAL (A.), 1997, Jérusalem : Destin d'une métropole, Paris : L'Harmattan (Comprendre le Moyen-Orient), pp. 199-200 ; 213-215 et les éditions du 27 novembre 2000 du Jerusalem Post et du 28 novembre 2000 de Ha'Aretz english edition.

27 Les nouvelles limités définies pour la municipalité de Jérusalem ont été largement étendues au-delà de celles de la municipalité de la Jérusalem-Est d'avant 1967. Comme le souligne V. Rivière-Tencer et A. Attal, les limites de la Jérusalem « réunifiée » dépassent nettement les $6 \mathrm{~km}^{2}$ que comptait Jérusalem-Est puisque ce sont plus de $70 \mathrm{~km}^{2}$ qui sont intégrés pour couvrir au total $108 \mathrm{~km}^{2}$, soit une superficie équivalente à celle de la ville de Paris (1997: 215). 
Dans un premier temps, parallèlement à l'aménagement du quartier juif au pied du Mur des Lamentations au cœur même de la vieille ville ${ }^{28}$, de nouveaux quartiers sont érigés dans l'idée notamment de rétablir une continuité territoriale avec le mont Scopus - où avait été implantée la première université juive de Jérusalem séparée du reste de la partie juive depuis la partition de 1949. Ainsi, selon les orientations données par le premier maire de Jérusalem, Teddy Kollek, « un pont terrestre » fut établi avec le mont Scopus par la création des quartiers de Ramat Eshkol, Givat ha-Mivtar et Givat Shapira (Dieckhoff, $1987: 129$; Rivière-Tencer, 1997 : 239).

À ces premières opérations d'urbanisme, a succédé, dès 1970 , une politique de consolidation des «positions juives » dans l'Est de la ville. Au Nord et à l'Ouest, les quartiers de Neve Yaacov et Ramot Allon ont été érigés tandis qu'à l'Est et au Sud, ceux de Talpiot-Est et Gilo voyaient également le jour. Ainsi, minutieusement, les limites de la Jérusalem « réunifiée » ont été dessinées dans l'idée d'accroître au maximum la superficie de la municipalité tout en incluant un minimum de villages arabes (Dieckhoff, 1987). Les no man's land laissés par ce « découpage ethnique » de la ville ont été comblés, si nécessaire après confiscation des terres arabes, par de nouveaux quartiers comme celui de Pisgat Zeev (1980) construit au Sud de Neve Yaacov (Rivière-Tencer, 1997 ; Kaminker, 1997).

En se dotant d'un tel programme de maîtrise territoriale de Jérusalem, les gouvernants israéliens sont entrés dans une réelle « bataille démographique » où l'immigration a pris une valeur cardinale.

Il s'agissait tout à la fois de rééquilibrer le solde migratoire de la ville (qui se tenait en moyenne à - 6000 hab. par an) et d'inverser la tendance démographique qui voyait la part de la population juive se réduire depuis 1967 . Pour atteindre ces buts, le renouveau migratoire des années quatre-vingt-dix a constitué un potentiel démographique de premier ordre.

Globalement, l'immigration d'ex-URSS n'a que moyennement concerné le district de Jérusalem et par conséquent, la ville en elle-même. À l'échelle de la ville « réunifiée », 24700 immigrants d'ex-URSS étaient enregistrés à Jérusalem en 1995, ce qui ne représentait que $5,8 \%$ de la population juive totale de la ville et aujourd'hui cette proportion s'établit à un niveau encore inférieur, environ $4,5 \%$ (soit un effectif ex-soviétique de 31 850). Cependant dans le contexte de perte de population dans lequel se tient Jérusalem depuis le début des années quatre-vingt, l'arrivée des exSoviétiques a compensé, en partie, le déficit migratoire. Sur la période 1989-1995, le gain brut de population juive a été de 59400 personnes, avec une part relative de l'immigration d'ex-URSS à hauteur de 41,5\%. Le soutien apporté par l'immigration est en cela non négligeable même si la croissance brute de la population juive de la Jérusalem « réunifiée » n'a pas été très supérieure à celle enregistrée sur la période 1983-1989, au cours de laquelle aucune vague migratoire importante n'avait intéressé

28 Voir notamment l'article de Michael Dumper, «Israeli settlement in the Old City of Jerusalem », Journal of Palestine Studies, vol.XXI, Number 4, Summer 1992, pp. 32-53. 
Israël (+ 4200 personnes, cf. tableau 1). Depuis, la tendance est restée la même avec une croissance inférieure à 20000 sur la période récente de l'immigration (1995-1998). Ces résultats confirment l'idée d'un amenuisement des capacités de Jérusalem à retenir sa population (DellaPergola, 1999 ; Rivière-Tencer, 2000) ${ }^{29}$.

Tableau 1 : Populations totale et juive de Jérusalem (1983-1998)

\section{Illustration non autorisée à la diffusion}

Source : Central Bureau of Statistics, Jerusalem, 2000.

À cette échelle d'analyse, il convient donc de conclure que l'immigration d'ex-URSS n'a pas profondément modifié la dynamique de croissance de la ville. La part de la population arabe n'a cessé de croître depuis la conquête de la partie orientale en 1967 passant de $25 \%$ à cette date à $32 \%$ aujourd'hui (cf. tableau 2).

Tableau 2 : Part des populations juive et « non-juive » à Jérusalem (1967-1999)

Illustration non autorisée à la diffusion

Source : M. DUMPER, 1997, The Politics of Jerusalem since 1967, NY, Columbia University Press, p. 77 et Ha'aretz English internet edition, May 4, 2000.

Si nous portons l'analyse de la croissance démographique dans sa dimension géostratégique, il faut toutefois souligner que l'immigration a favorisé la réalisation des « objectifs israéliens », à savoir l'établissement d'une majorité juive dans la partie orientale de la ville au début des années quatre-vingt-dix. En 1993, les données publiées ont révélé le basculement de la majorité démographique en faveur de la population juive et ce changement a reposé, en partie, sur l'immigration d'ex-URSS. Fin 1995, les nouveaux quartiers juifs de Jérusalem-Est avait en effet accueilli plus de 10500 ex-Soviétiques, soit près de $45 \%$ des immigrants « russes » de la ville. Au Sud de la partie orientale de la ville, les quartiers de Gilo et de Talpiyot Est ont été les principaux lieux d'installation des immigrants tandis qu'au Nord, les quartiers de Ramot Alon (Nord) mais surtout ceux de Pisgat Zeev et Neve Ya'acov ont rassemblé près de 7000 ex-Soviétiques.

29 Pour 1998, la balance migratoire de Jérusalem a été déficitaire de 10400. 
Cependant, le tassement de la croissance brute de la population juive de Jérusalem et la consolidation des filières migratoires ex-soviétiques vers les villes de Haïfa, Ashdod, Be'er Sheva et même Bat Yam, n'ont pas permis à la population juive de conserver la majorité acquise sur les quartiers Est de la ville. Selon de récentes données diffusées par Peace $N_{0 w}{ }^{30}$, on y compterait aujourd'hui 181000 Arabes et 156 662 Juifs.

Une telle dynamique n'a fait qu'affaiblir les «prétentions démographiques » visant à l'établissement d'une "majorité juive à l'échelle du Grand Jérusalem » (cf. carte 3) même si les colonies de Cisjordanie ont connu un essor de population non négligeable avec cette vague migratoire. Hormis Ma'ale Adoumim, qui a accueilli plus de 3200 ex-Soviétiques sur la période, le «Grand Jérusalem » n'en a vu s'installer qu'un peu plus de 1200 , principalement à Pisgat Zeev; soit l'équivalent d'environ $8 \%$ du contingent d'ex-Soviétiques au-delà de la ligne verte

Lors de l'allocution où il avait souligné la nécessité d'un « Grand Israël », Yitshak Shamir avait déclaré : «Autour de nous les Arabes sont en plein désarroi, en état de panique [...] ; ils sont submergés par un sentiment de défaite, parce qu'ils voient que l'Intifada ne sert à rien; ils ne peuvent arrêter le flot naturel du peuple juif vers sa patrie - et, après tout, c'est bien ce autour de quoi tourne le conflit ${ }^{31}$. Aujourd'hui, ces propos prennent la forme d'une véritable erreur d'analyse. L'immigration d'ex-URSS a certes signifié la persistance d'un lien étroit entre la diaspora et Israël - même si certains soulignent que le flot migratoire repose sur l'absence de réelles alternatives à l'aliya - mais elle n'a en aucun cas été l'acteur de l'établissement d'une « suprématie démographique pour un Grand Israël » et encore moins, celui d'un renoncement du peuple palestinien à clamer ses droits, comme en témoigne l'éveil d'une seconde Intifada.

L'immigration a contribué à l'accroissement démographique dans les territoires conquis en 1967, principalement à Ariel et Ma'ale Adoumim, mais, globalement, à un niveau moindre que la dynamique générale de colonisation. Les exSoviétiques qui résident en Cisjordanie et à Gaza, ne représentent que $7,5 \%$ de la présence juive dans ces territoires et la géographie de cette immigration au-delà de la ligne verte apparaît véritablement comme une géographie par défaut. Il s'agit d'une mise en lumière de la politique du fait accompli, développée avec plus ou moins d'intensité sous les successives administrations israéliennes. Aujourd'hui, la politique qui a consisté à mettre à disposition des logements à Jérusalem-Est ou en Cisjordanie, selon l'idée que là où résideront des juifs se trouvera le tracé des frontières avec un futur État palestinien, se révèle être une impasse, car maintenir une telle position ne peut aboutir qu'à accroître l'intensité du conflit. Les négociations territoriales dans le cadre du processus de paix passeront nécessairement par le retrait d'une partie des colonies et les répercussions sociales de ce retrait ne manqueront pas.

30 Ha' aretz English internet edition, May 4, 2000.

31 Le Monde daté du 16 janvier 1990.

REMI 2000 (16) 3 pp. $201-218$ 
Pour conclure, je retiendrais ce dernier point car il est par trop souvent occulté par le débat suscité par les deux premiers. En qualifiant la présence ex-soviétique dans les territoires occupés de géographie par défaut, nous rappelions également que c'est sous le coup de difficultés économiques que les nouveaux immigrants ont été « attirés par les lueurs des colonies », mais, ils n'ont pas été les seuls. En effet, la saturation du marché immobilier et plus globalement l'augmentation du coût de la vie ont contribué à repousser au-delà de la ligne verte de nombreux jeunes couples aux revenus trop bas pour résister à l'offre d'une résidence. Aujourd'hui, les événements rendent leur futur incertain et ne font qu'accroître la radicalisation des rapports sociaux au sein même d'Israël.

\section{Références bibliographiques}

BENSIMON (D.), ERRERA (E.), Israéliens, des Juifs et des Arabes, Paris, Complexe, 1989.

BERTHOMIERE (W.), « Analyse de l'immigration récente en Israël » in SANGUIN (A.-L.), Mare Nostrum, dynamiques et mutations géopolitiques de la Méditerranée, L'Harmattan (Géographie et cultures), 2000.

BERTHOMIERE (W.), «L'épopée des Juifs d'ex-URSS en Israël », Confluences Méditerranée, $\mathrm{n}^{\circ} 26,1998$.

COURBAGE (Y.), « Redistributions des cartes démographiques au Proche-Orient. L'avenir du peuplement de la région Israël-Palestine », Revue d'études palestiniennes, ${ }^{\circ} 18$ (ns), 1999.

COURBAGE (Y.), « La fécondité palestinienne des lendemains de l'Intifada », Population, $\mathbf{n}^{\circ} 1$, 1997.

COURBAGE (Y.), « Contrastes démographiques en Israël : tendances récentes », Population, $\mathrm{n}^{\circ} 3,1993$.

DELLAPERGOLA (S.), «A new look at the Jerusalem's future population : demography, multiculturalism and urban planning ", communication faite à la European Population Conference, The Hague, The Netherlands, 1999.

DIECKHOFF (A.), Israéliens et Palestiniens. L'épreuve de la paix, Paris, Aubier, 1996.

DIECKHOFF (A.), Les espaces d'Israël, Paris, FEDN (Les 7 épées), 1987.

DUMPER (M.), The politics of Jerusalem since 1967, New York, Columbia University Press (IPS Series), 1997.

FARGUES (P.), « Les données démographiques de la paix au Proche-Orient » in BLIN (L.) et FARGUES (P.), L'économie de la paix au Proche-Orient, Tome 2, Paris, Maisonneuve et Larose-CEDEJ, 1995.

ISRAEL CENTRAL BUREAU OF STATISTICS, Statistical Abstract of Israel 2000.

ISRAEL CENTRAL BUREAU OF STATISTICS, Immigrant Population from USSR (Former), selected data 1998, Jerusalem, 2000.

ISRAEL CENTRAL BUREAU OF STATISTICS, Immigrant Population from Former Soviet Union, 1995 : Demographic Trends, Jerusalem, 1998.

KAMINKER (S.), « Jérusalem-Est, un cas d'école en matière de planification politique », in DHOQUOIS-COHEN (R.) et al. (dir.), Jérusalem, ville ouverte, Paris, L'Harmattan (Les Cahiers de Confluences), 1997.

KAPELIOUK (A.), Israël : la fin des mythes, Paris, Albin Michel, 1975.

NEWMAN (D.), Population, settlement and conflict : Israel and the West Bank, Cambridge : Cambridge University Press (Update), 1992. 
RIVIERE-TENCER (V.), ATTAL (A.), « Jérusalem, une paix pour demain ? 》 in CHAGNOLlaUd (J.-P.) et al., Palestiniens et Israéliens, le moment de vérité, Paris, L'harmattan (les cahiers de Confluences), 2000.

RIVIERE-TENCER (V.), ATTAL (A.), Jérusalem : Destin d'une métropole, Paris, L'Harmattan (Comprendre le Moyen-Orient), 1997. 